\title{
Evolución del marketing digital: caso de la marca ecuatoriana Forestea
}

\author{
Evolution of digital marketing: \\ case of the ecuadorian brand Forestea
}

\author{
Diego Bravo Torres \\ Universidad Nacional de Loja, Ecuador \\ dabravot@unl.edu.ec \\ https://orcid.org/0000-0002-6347-8752 \\ Mónica Hinojosa-Becerra \\ Universidad Nacional de Loja, Ecuador \\ monica.hinojosa@unl.edu.ec \\ http://orcid.org/0000-0003-3288-2135
}

Recibido: 04/04/2021 Revisado: 15/05/2021 Aceptado: 29/05/2021 Publicado: 01/09/2021

\begin{abstract}
Resumen
Se analizó las estrategias del marketing digital de la marca Forestea en la red social Facebook con el objetivo establecer si existe una evolución en el desarrollo digital de la marca. Forestea lanzó su marca en Facebook en 2013 como una estrategia para diversificar sus consumidores. Se analizaron los productos incluyendo: composición, factores cromáticos, tipografía, mensaje, contenido y forma de difusión. Se observó la interacción de los seguidores con su página web de Facebook. Los datos obtenidos se sistematizaron en fichas de observación. Esto permitió determinar las herramientas y recursos que Forestea ha utilizado para fortalecer su marketing digital a través de Facebook. Se contabilizó los productos publicados en la red social, los meses de enero, junio y diciembre de los años 2013 a 2018; debido a que las ventas del producto tienen un incremento del $45 \%$ en el mercado nacional e internacional en enero. En diciembre desciende un $50 \%$ y en junio se mantienen equilibradas. Los datos almacenados se resumieron en tablas por año, con un total de 24 fichas de observación durante toda la investigación. Desde 2013, el marketing digital de la marca ha evolucionado cambiando los colores la tipografía y la forma de presentar las imágenes en la red social. Pero aún necesitan afianzar la credibilidad de los seguidores y fidelizarlos a la marca.
\end{abstract}

\section{Palabras clave}

Comunicación, marketing, Facebook, Forestea, marca, digital, Internet, fidelización.

Forma sugerida de citar: Bravo, D., \& Hinojosa-Becerra, M. (2021). Evolución del marketing digital: caso de la marca ecuatoriana Forestea. Universitas-XXI, 35, pp. 61-81. https://doi.org/10.17163/uni.n35.2021.03 


\begin{abstract}
The digital marketing strategies of the Forestea brand were analyzed on the Facebook social network in order to establish whether there is an evolution in the digital development of the brand. Forestea launched its brand on Facebook in 2013 as a strategy to diversify its consumers. The products were analyzed including: composition, color factors, typography, message, content and form of diffusion. Followers' interaction with their Facebook web page was observed. The data obtained were systematized in observation files. This made it possible to determine the tools and resources that Forestea has used to strengthen its digital marketing through Facebook. The products published on the social network were counted, the months of January, June and December from the years 2013 to 2018; Due to the fact that the sales of the product have an increase of $45 \%$ in the national and international market in the month of January. In December it falls by $50 \%$ and in June they remain balanced. The stored data were summarized in tables per year, with a total of 24 observation files throughout the investigation. Since 2013, the brand's digital marketing has evolved by changing colors, typography and the way images are presented on the social network. But they still need to strengthen the credibility of the followers and retain them to the brand.
\end{abstract}

\title{
Keywords
}

Communication, marketing, Facebook, Forestea, brand, digital, Internet, loyalty.

\section{Introducción}

Las redes sociales han significado un cambio en los procesos comunicativos. Representan un espacio abierto que permite a los internautas exponer de forma abierta sus puntos de vista (Broun-Isaac, 2020). Compartir es el objetivo de las redes sociales. También permite hacer contactos profesionales y a las empresas les ha abierto una puerta para relacionarse con posibles consumidores (Gómez-Carreño \& Palacios-Alvarado, 2021).

Debido a la gran demanda de usuarios en redes sociales los medios tradicionales se han visto desplazados por las mismas (Matassi \& Boczkowski, 2020). Han aparecido nuevas formas de promocionar productos o servicios a través de redes sociales (Filippone et al., 2021). Algo que las empresas han encontrado muy beneficioso, ya que las redes sociales brindan un amplio mercado para promocionar tanto su imagen como sus productos (Piatykop $\&$ Pronina, 2020). En estos casos el índice de influencia no depende de las empresas sino también de la predisposición de las personas en las redes so- 
ciales a ser influenciadas (Lara-Navarra et al., 2018). Las empresas de todo tipo, así como multinacionales de bebidas, pequeñas y medianas empresas, se ven obligadas a introducirse en el mundo digital (Tarro et al., 2017).

Alcanzar el éxito a través de redes sociales no solo depende de tener presencia en las mismas. Obedece a distintos factores: generar clientes en redes sociales, desarrollar estrategias digitales para promocionarse, llegar a públicos específicos, fidelizar clientes, generar campañas creativas y lograr beneficios para la empresa (López-Jáuregui et al., 2019; Ballestar et al., 2019).

Actualmente las empresas de bebidas están batallando contra las multinacionales que acaparan el mercado con sus productos, llegando a tener ventas a escala mundial. Este es el caso de Coca-Cola, marca que ha ganado posicionamiento en el mercado y ha tenido que adaptarse a las nuevas formas de desarrollo de marketing digital en las redes sociales con sus campañas publicitarias (Boelsen-Robinson et al., 2016; Tran, 2021; Berceruelo et al., 2017). Este es uno de los retos para empresas como Forestea que busca alcanzar éxito empresarial y generar productos comunicativos a través de redes sociales para poder generar un posicionamiento empresarial marcado (Jumbo, 2019).

Esta investigación se basa en determinar cuáles son las estrategias publicitarias de la empresa de bebidas Induloja, con su producto Forestea en Facebook y cuál es el grado de interactividad que consigue con sus seguidores. Este tipo de investigaciones, concretamente a través de Facebook, actualmente se están desarrollando para determinar la competitividad de las empresas (AltamiranoBenítez et al., 2018). Se han realizado entrevistas a expertos y desarrollado fichas de observación que permitieron llevar a cabo el análisis sobre el tema.

Las fichas de observación se realizaron con el fin de analizar las estrategias publicitarias de la marca Forestea en Facebook utilizadas durante el período de la investigación, que son seis años (2013-2018). Los objetivos específicos son analizar los productos comunicacionales de la empresa Forestea, desde sus inicios en 2013 hasta diciembre de 2018 en Facebook y determinar las herramientas, recursos técnicos, estrategias y métodos que la marca Forestea utiliza para el posicionamiento de su imagen en esta red social.

\section{La comunicación y el marketing}

Internet ha permitido compartir los productos e información a través de las redes sociales. Las empresas, instituciones y organizaciones se han adapta- 
do a las formas de comunicación con sus públicos tanto internos, como externos. Las redes sociales son una herramienta primordial para hacer marketing, generando la idea de que, si hoy la empresa no usa las redes sociales estaría dejando de lado su éxito, poniéndose de cara al fracaso (Linares-Cazola \& Pozzo- Rezcala, 2018, p. 158). Con esto se han creado y adaptado estrategias digitales de comunicación para segmentar o diversificar sus públicos.

El marketing permite a las empresas conocer sus públicos y crear estrategias de ventas y consumo de servicios. El marketing gracias a las redes sociales se ha convertido en un aspecto fundamental para las empresas a la hora de comunicarse con sus clientes (Jiménez-Marín \& Pérez-Curiel, 2021, p. 17). Podemos entender que la comunicación no solo informa, debe llegar al consumidor generando una reacción; "en este sentido, el proceso debería concluir con la obtención de una respuesta favorable del destinatario del mensaje, que en último término consiste en la compra o no compra del producto" (Monferrer, 2013, p.152).

Esta premisa de Monferrer (2013) se argumenta con los pensamientos de Cardozo- Valesca (2007, p.199) que afirma que: "la comunicación puede ser considerada como un medio para motivar, persuadir, convencer, comprometer ideas, facilitar procesos, armonizar puntos de vista".

El objetivo es inducir una idea de aceptación del mensaje receptado. Este es el resultado que el marketing busca para sus clientes. El marketing abarca un proceso en el que las compañías establecen relaciones con sus potenciales clientes, con la finalidad de recibir una retribución por parte del consumidor. Comprende un intercambio de valores entre vendedor y consumidor, una situación en la que ambos generan beneficios. Estas definiciones coinciden con los teóricos Kotler y Armstrong (2003,p. 4) quienes afirman que: "La meta doble del marketing consiste, por un lado, en atraer a nuevos clientes prometiéndoles un valor superior y, por otro, mantener y hacer crecer a los clientes actuales al entregarles satisfacción".

El desarrollo del marketing se da cada vez que una empresa genere un intercambio de valor con otra unidad social. Es por esto que la finalidad del marketing es el intercambio (Monferrer, 2013). Dicho intercambio permite un proceso donde ambas partes finalizan el suceso con una transacción. Lo que se traduce que la empresa intercambia su producto o servicio por una satisfacción por parte del cliente, convirtiéndose en un ciclo repetitivo, donde pueden intervenir otros factores, económicos o sociales. El uso de redes 
sociales como herramienta de marketing ha aumentado en los últimos años (Jiménez-Marín \& Pérez-Curiel, 2021, p. 14).

Con la aparición de Internet se ha logrado mayor facilidad en la interacción y la comunicación de las personas a tal punto que ya no es necesaria la comunicación en un medio físico. Dentro de este escenario entra el marketing digital. Selman (2017) lo define como las estrategias de ventas creadas desde un sitio web. Lo importante es lograr que el usuario entre a la página, recorra y concluya la acción de compra o selección de un servicio.

Una herramienta que ha contribuido a la evolución del marketing digital es la Web 2.0. Es una plataforma que contiene datos generados por los usuarios que permite transmitir "publicidad viral a muchas personas de forma individualizada y que a través de las redes sociales contribuye a difundir mensajes con fines privados o publicitarios entre los usuarios" (García, 2011, p. 41).

Con herramientas como la Web 2.0 o la Web 3.0:

La mercadotecnia en Internet se está revelando como una herramienta eficiente para segmentar mercados y conocer mejor a los clientes para lograr su fidelidad, generándole a las empresas la posibilidad de conseguir una relación más directa con sus clientes. (Camejo et al., 2020, p. 82)

Las redes sociales han trascendido la interacción personal para abrir paso a un espacio de interacción empresarial, debido al fácil acceso, bajo costo, y su alcance, que es mayor al de los medios de comunicación convencionales. A estos perfiles de redes sociales en los que están inmersos organizaciones o empresas, Hütt-Herrera (2012, p. 124) los denomina "redes profesionales" que tienen la facilidad de desarrollar una amplia lista de contactos profesionales para mantener accesible la comunicación, interacción e intercambios comerciales entre las personas y donde los usuarios pueden comunicarse entre sí, de acuerdo con sus necesidades, gustos, expectativas y preferencias.

Actualmente Facebook es una de las redes sociales más usadas entre los internautas y su popularidad se debe a la facilidad para comunicarse e interactuar con otras personas alrededor del mundo. Las empresas han encontrado en esta red social un espacio que les permite posicionar sus marcas, a través del contacto directo con los posibles consumidores (Jackler et al., 2019; Pérez-Dasilva et al., 2013; Gálvez-Clavijo, 2016; Zeler-Pighin, 2017).

Las grandes y medianas empresas de Latinoamérica mantienen una identidad digital consolidada en sus redes sociales. Sforzin (2016) conside- 
ra que el acceso a Internet es desigual entre los hogares más ricos y los más pobres de Latinoamérica. Un 60\% de empresas de Ecuador que han designado a Facebook como una de las más utilizadas y dándole el primer lugar (Jiménez, 2016, p. 306). Para Boyeras et al. (2019) las interacciones en Facebook pasaron del "me gusta", como reacción única, a incorporarse a partir de 2016 "me encanta", "me importa", "me divierte", "me asombra", "me entristece", "me enoja", a una seria de emojis y opciones de compartir, como chat, creación de grupo y opciones empresariales.

\section{La empresa Forestea}

En 2013 hicieron el lanzamiento al público local de la bebida Forestea. El lema con el que inició la comercialización de este producto es brindar al sector local, nacional e internacional una bebida sana, de buen sabor y que contribuya a la salud de los consumidores. De allí que su misión sea: "Producir y entregar a nuestros consumidores alimentos nutritivos, naturales y sanos, aportando positivamente a la sociedad en su salud y bienestar". Y se planteen como visión ser:

Una empresa joven cuyos esfuerzos se orientan a la innovación en la industria de alimentos. Nuestro objetivo es cumplir con las exigencias de una sociedad que prefiere los productos saludables y socialmente responsables, creando un compromiso con nuestros accionistas, colaboradores y consumidores. (http://www.forestea.com.ec/)

En el transcurso de cinco años la empresa Induloja ha logrado ingresar el producto Forestea en mercados como Estados Unidos, Chile y Colombia. Producen cinco sabores diferentes con base en el producto original. Actualmente distribuyen: Forestea Horchata, Forestea Mandarina, Forestea FresaLimón, Forestea Manzana Verde y Forestea Frutos Tropicales.

Forestea nació en el 2011 de un concurso de emprendimientos y nuevas ideas de negocios, convocado para la Zona 7 de Ecuador por la Incubadora de Emprendimientos Prendho de la Universidad Técnica Particular de Loja (UTPL) y el Ministerio de Industrias y Productividad (MIPRO).

Los resultados del estudio de mercado permitieron la creación de la fórmula de la horchata Forestea, que se patentó después de un año y medio de su creación, en la Agencia Nacional de Regulación, Control y Vigilancia 
Sanitaria (ARCSA). Según explican los fundadores de la empresa se tardó este tiempo debido a que al ser Forestea una fórmula nueva y no existir una patente que incluya componentes similares, se debía probar las propiedades medicinales del producto. Por tal razón el estudio de los componentes químicos de las hierbas les tomó más tiempo de lo previsto. La horchata es una bebida tradicional ecuatoriana (Armijos et al., 2020) con propiedades medicinales (Guevara et al., 2020; Guevara et al., 2019; Ríos et al., 2017).

\section{Imagen 1}

\section{Sabores de Forestea}

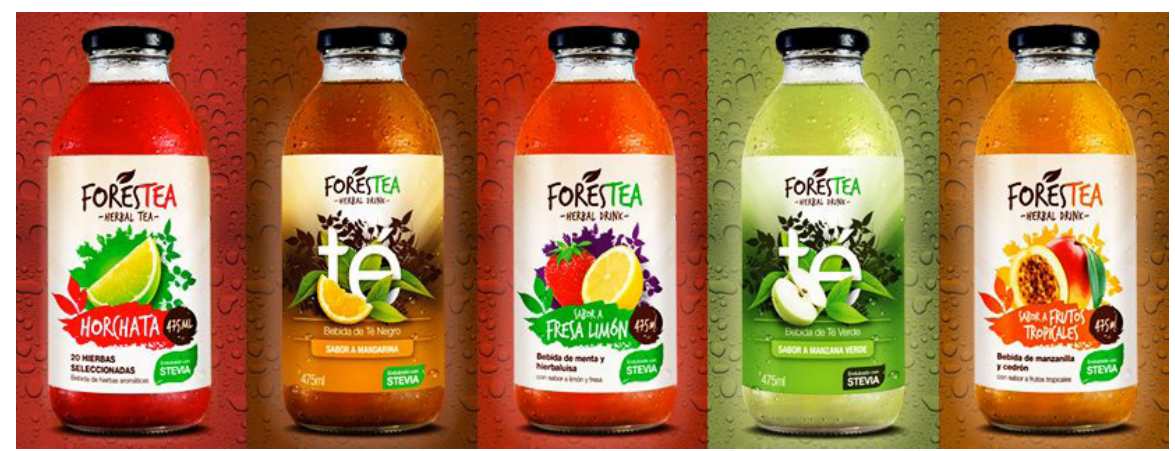

Fuente: https://www.facebook.com/foresteaecu

Forestea salió al mercado en el 2013. En sus inicios se comercializó como un producto realizado artesanalmente, explica Santiago Torres, gerente de la empresa, "nosotros éramos nuestra propia fuerza de ventas y distribución". En el 2015 se asociaron con Family Foods en una alianza estratégica con distribuidores en Estados Unidos, como resultado de esta alianza, la bebida empezó a ser comercializada en los Estados Unidos.

La COPADE (Comercio para el Desarrollo), en el 2018, reconoció a Forestea por cumplir los requisitos establecidos por la World Fair Trade Organization (Organización Mundial de Comercio Justo), como una empresa que ejerce el comercio justo, responsable y equitativo. Aval que hace de Forestea una empresa socialmente responsable.

Las estrategias publicitarias de Forestea reflejan una evolución en el marketing digital. Esto se refleja en el número de seguidores que Forestea ha venido acumulando a lo largo de estos años. Sobre todo en Facebook, 
donde mantiene una cantidad de 45000 seguidores, alrededor de 4000 seguidores en Instagram y un aproximado de 400 seguidores en Twitter. La estrategia digital de la marca se ha enfocado en Facebook donde acumula la mayor cantidad de seguidores.

La empresa Forestea publica actualmente, en el 2021, un promedio de una publicación cada dos días en Facebook. Existen ocasiones en las que las publicaciones son diarias incluso llegando a publicar dos post por día. Forestea al mes genera un aproximado de 25 a 35 publicaciones.

\section{Metodología}

La investigación fue realizada con base en los postulados de los métodos cuantitativo y cualitativo, metodología que trata de "identificar la naturaleza profunda de las realidades, su sistema de relaciones, y su estructura dinámica" (Pita-Fernández \& Pértegas-Díaz, 2002, p. 76). Trata de determinar todos los factores que influyen en una realidad para poder analizar su comportamiento con todos los elementos que intervienen. La metodología cualitativa ayuda para analizar los datos y variables observables presentes en la realidad de la empresa Forestea y así definir conclusiones con base en los resultados obtenidos.

La metodología cualitativa es definida como "la investigación que produce datos descriptivos, tales como: las propias palabras de las personas, habladas o escritas, y la conducta observable" (Castaño-Garrido \& Quecedo-Lecanda, 2003, p. 7). La investigación cualitativa contribuye a la culminación satisfactoria de los objetivos planteados al inicio de la investigación (Marín-Gutiérrez et al., 2016).

Se utilizó este método para lograr obtener los datos teóricos y nuevos conocimientos que permitan desarrollar la investigación. Se logró determinar los pasos e instrumentos necesarios en la investigación propuesta, tomando en cuenta los objetivos planteados. El método cualitativo se ha usado para describir los hechos, y situaciones que se observaron durante la investigación y el período de estudios. Se pudo describir el comportamiento o estado de las variables de la investigación, específicamente en los resultados observables del análisis de los productos comunicativos presentes en Facebook. La aplicación del método cualitativo contribuyó para la elaboración 
de las fichas de observación, los resultados y las conclusiones (Marín-Gutiérrez, 2020).

Como parte del método cualitativo se tomaron las fuentes de información tales como textos, libros, revistas y páginas web especializadas. La elaboración de una revisión de fuentes de información "pone a prueba la capacidad de juicio y las competencias de lectura y escritura de los estudiantes" (Peña, 2010, p. 1). Se realizó una lectura comprensiva y analítica de los textos obtenidos. Analizamos las publicaciones realizadas por Forestea en Facebook desde el 2013 al 2018. Para ello segmentamos la muestra tres meses por año. Los productos comunicacionales analizados corresponden a enero, junio y diciembre de cada año, desde el 2013 al 2018, presentes en Facebook en su página oficial de Forestea (https://www.facebook. com/foresteaecu).

También se utilizó la técnica cuantitativa a través de una ficha de observación que se tenía que ir cumplimentando por cada anuncio en Facebook analizado. Para poder realizar el análisis se elaboró una primera ficha en la que se contabilizó la cantidad de productos publicados en Facebook. El conteo se realizó a productos publicados en enero, junio y diciembre de 2013, 2014, 2015, 2016, 2017 y 2018. Se evidenció que las ventas del producto tienen un incremento del $45 \%$ en el mercado nacional e internacional en el mes de enero. En diciembre las ventas descienden un $50 \%$. Y en junio los porcentajes de las ventas se mantienen equilibradas. Esto nos permite evaluar las publicaciones e imágenes que se comparten en meses en los que la producción asciende, desciende o se mantiene.

Los datos almacenados se resumieron en cuatro tablas por año. Resultaron un total de 24 fichas de observación durante toda la investigación. El 25 de julio de 2013, Forestea inició su estrategia de marketing digital y hasta el 2018 había realizado un total de 831 anuncios en su cuenta de Facebook. Por lo tanto procedimos a calcular la muestra que necesaria. El universo es 831 publicaciones, la heterogeneidad fue del $50 \%$, el margen de error fue del $\pm 5 \%$ y el nivel de confianza fue del $90 \%$. El resultado fue de analizar 205 publicaciones durante los seis años. Se aplicó las fichas de observación a una muestra de 209 publicaciones (cuatro más de las necesarias), dando como resultado la Tabla 1. 
Tabla 1

Total de publicaciones en Facebook de la cuenta de Forestea

\begin{tabular}{|l|c|c|}
\hline \multicolumn{1}{|c|}{ Año } & Muestra & Cantidad total \\
\hline 2013 & 8 & 32 \\
\hline 2014 & 7 & 28 \\
\hline 2015 & 4 & 16 \\
\hline 2016 & 67 & 263 \\
\hline 2017 & 77 & 308 \\
\hline 2018 & 46 & 184 \\
\hline Total 209 & & 831 \\
\hline
\end{tabular}

Fuente: Elaboración propia.

\section{Entrevistas}

La entrevista es una técnica que sirve en la investigación cualitativa para poder obtener datos y realidades necesarias para el desarrollo de la investigación. "se define como una conversación que se propone un fin determinado distinto al simple hecho de conversar" (Días et al., 2013, p. 163). Se busca que la información obtenida mediante esta técnica sea la más precisa posible para luego transcribirla e interpretarla fielmente a la realidad.

El tipo de entrevista que se realizó se categoriza como entrevista semiestructurada, que "ofrece un grado de flexibilidad aceptable, a la vez que mantiene la suficiente uniformidad para alcanzar interpretaciones acordes con los propósitos del estudio" (Días et al., 2013, p. 163). Las entrevistas semiestructuradas se basan en preguntas planeadas que pueden adaptarse a los entrevistados y de esta manera aclarar cuestiones que no hayan quedado evidentes.

Se entrevistó a Santiago Torres, gerente general de Forestea, quien colaboró con información sobre la historia y creación de la bebida Forestea. La entrevista sirvió para tener una idea de cómo es el manejo de las redes sociales de Forestea. Se realizó una entrevista a Francisco Vicuña, coordinador de la incubadora Prendho UTPL, quienes apoyaron a que la empresa pudiera ser creada y creciera de la manera en que lo ha venido haciendo. Ayudó a despejar dudas acerca del apoyo brindado a los emprendedores para la creación de Forestea, y también sirvió para contrastar y comparar los datos obtenidos de la entrevista con Santiago Torres. 


\section{Resultados}

En el 2013 se realizó un total de ocho publicaciones. De ellas cinco fueron únicamente texto y tres contenían imagen. Cuatro publicaciones promocionaron el producto y las otras cuatro hacían mención a la marca. En los productos comunicacionales de Forestea aparecen los colores rojos, negro, verde y blanco de forma alternada en las ocho publicaciones, en una se encuentra el azul. El logo de la marca aparece tres veces ubicado a la derecha y una a la izquierda y una en el centro del cuadro. De las seis publicaciones que tienen texto, en tres la tipografía varía entre los colores negro y rojo. La ubicación del logotipo utilizado en tres publicaciones se colocó en el centro del producto y en la parte superior izquierda del mismo. Lo mismo pasa con la ubicación del producto. En las piezas comunicacionales se ubicó al producto en la parte superior en dos ocasiones, y la parte central en una ocasión.

Cuatro publicaciones mantuvieron el mensaje directo de consumir la bebida Forestea. El único hashtag utilizado fue: \#DescansaChucho, en referencia al fallecimiento del jugador de futbol ecuatoriano Christian Benítez. Los colores más usados fueron el rojo, negro, verde y blanco. En cuanto al nivel de respuesta por parte de los seguidores se obtuvo que todas las publicaciones alcanzaron la cantidad de 133 "me gusta", y un total de siete comentarios. El primer año la estrategia digital fue realizada por los dueños de la empresa. Esto se evidencia en la baja cantidad de interacción de los seguidores de la empresa.

En el 2014 se analizaron siete productos en Facebook, de los cuales seis fueron imágenes propias y solamente un producto fue compartido de otra página. Se utilizaron siete hashtags diferentes durante los tres meses del período de estudio. Los cuales fueron: \#SUPERMAXI \#TIA \#FIBECA \#MegustaForestea \#Forestea \#Naturaleza \#ForesteaConLaSeleccion. Los mensajes fueron direccionados al consumo de la bebida. Y en la cantidad de interacciones se logró la cantidad de 1104 "me gusta" y un comentario positivo.

En la muestra de 2014 se puede notar que el tipo de contenido que se empleó en todos los productos fueron imágenes tratadas digitalmente. La mayoría de productos son de propia autoría con una publicación compartida. En cuanto al tipo de mensaje que se presentó en la muestra se evidencia que la bebida tuvo mayor peso, junto con temas festivos como el tema de la Navidad. Se utilizó la época navideña para promocionar a los productos y llegar a más personas. En el caso de los colores utilizados destacan el color 
rojo, verde y blanco. Colores que identifican a la marca. A los que sigue el amarillo, azul y el negro.

El punto de interés en el que se ubica el logotipo es en la parte superior izquierda del producto en tres ocasiones, en la parte centro superior en dos ocasiones y en la parte superior derecha en una ocasión, no se evidencia un criterio claro de la ubicación del logo. Lo mismo sucede con la ubicación del producto en el cuadro de la imagen en cuatro imágenes se los coloca en la parte inferior izquierda y en dos publicaciones en el centro, consideramos que esto se lo realiza con la intención que no interrumpa la visibilidad de todo el arte.

Dentro del uso de la tipografía, y su color, y con el análisis del gráfico, se puede determinar que la letra está ubicada en la parte central del afiche, en donde priman colores como el rojo en siete publicaciones, al que le sigue el negro, posteriormente el blanco. Aunque también se utilizaron colores como el verde y amarrillo, pero en mucha menor proporción.

En el 2015 las cuatro publicaciones analizadas fueron imágenes y se usó un hashtag: \#Forestea. Los mensajes de las publicaciones fueron direccionando al consumo de la bebida Forestea y se obtuvo una cantidad de 887 "me gusta", y un total de 24 comentarios; siete positivos y 17 comentarios neutros.

La mayoría de productos que se difundieron en todo el año fueron imágenes tratadas digitalmente. El producto Forestea tiene mayor protagonismo en el mensaje de las piezas publicitarias. Los colores rojo y blanco fueron los más usados para los productos publicitarios en tres de las cuatro publicaciones. Desde el inicio de la estrategia de marketing en Facebook estos colores han sido los que han primado en la paleta de utilizadas para las fotografías.

La ubicación del logotipo sigue siendo variable sea en la parte superior izquierda, superior derecha o en el centro del cuadro. También se utilizaron productos en los que no se ubicaba al logotipo en el post. En cuanto a la presencia de la bebida Forestea se puede determinar que se ubicó al producto en la parte inferior izquierda del afiche publicitario en tres ocasiones. Esto por generar mayor peso visual al contexto del mensaje, quitándole el protagonismo a la bebida.

La letra de color negro en las imágenes de los productos de Forestea fue la que predominó en la mayoría de los productos. Al que le siguen colores como el blanco y rojo, para luego, aparecer en menor proporción el azul y se incluye por primera vez el color marrón. Predomina el color negro se debe a que el fondo de la imagen tenía en mayor proporción el color blanco, algo que técnicamente sería una posible hipótesis de por qué se utilizaron estos colores. 
El 2016 fue un año de notable crecimiento en seguidores para Forestea, esto se evidencia en los datos obtenidos en las fichas de observación. En 2016 se analizaron 67 publicaciones, de las cuales 64 publicaciones fueron imágenes, y tres publicaciones fueron GIFS. Se utilizaron los siguientes hashtags: \#ActitudForestea, \#sientelatradicion, \#Forestealovers, \#CarrieFisher. El contexto del mensaje de las publicaciones es en su mayoría el consumo de la bebida y en este año se evidencia un notable crecimiento y evolución de la marca, llegando a más de 12628 "me gusta", 204 "me encanta", 36 "me divierte", 6 "me asombra", 12 "me entristece", 1 "me enoja" y acumulando la cantidad de 129 comentarios y 379 compartidas de las publicaciones. En el 2016 que se llegó a establecer una mayor cantidad de retroalimentación con el público.

El tema en el que el mensaje se basó para la difusión de contenidos fue el del producto. Un dato interesante en esta etapa es que ya se empieza a trabajar con mayor frecuencia las festividades como Navidad, Año Nuevo o días internacionales.

Todos los productos publicitarios tienen armonía en la composición en cuanto a colores, y se utilizó el rojo y azul, la mayor cantidad de veces. A estos primeros los continúan el verde, naranja y blanco. Aunque también se utilizaron colores como marrón, amarillo, negro, púrpura y gris, pero en menores proporciones. La marca no tiene un color establecido para las publicaciones sino que este varía dependiendo del contexto del mensaje del producto.

En cuanto a la ubicación del logotipo de la bebida Forestea, se puede determinar que la mayoría de veces se ubicó en la parte inferior izquierda en 53 afiches publicitarios. Podemos observar que se empieza a estandarizar la ubicación de los elementos en el cuadro de la imagen.

La tipografía de color blanco es la más utilizada en 22 publicaciones en cuanto a los productos comunicacionales de la marca. Se evidencia que aparecen el rojo, amarillo, azul, blanco, púrpura y negro, pero en cifras más bajas. Siendo la parte central en la que mayores veces se ubicó al texto en los productos publicitarios.

En el 2017 se analizaron 77 publicaciones de las cuales 67 fueron imágenes, seis fueron GIFS, y las cuatro restantes fueron videos de corta duración. Se utilizaron los siguientes hashtags: \#Actitudforestea, \#DeténElMundo, \#ChristmasFacts, \#PrideDay, \#DiamundialDelaEducacionAmbiental. El mensaje de las publicaciones tiene la misma intención de los años anteriores, invita al consumo de la bebida Forestea. En este año se obtuvo alrededor 
de 18113 "me gusta", 413 "me encanta", 6395 "me divierte", 47 "me asombra", 78 "me entristece" y 26 "me enoja". Llegando a ser el año con mayor interacción en la red social.

En el 2017 se encuentran diferencias sobre el contenido difundido. Aunque los productos más publicados siguen siendo las imágenes. En el 2017 también se utilizaron otro tipo de productos como videos, cuatro en total, que tienen un promedio de duración de 13,25 segundos.

Las temáticas tienen el mismo enfoque del año anterior, la bebida fue el mensaje del que más se habló en los productos publicados, a lo que siguen las festividades, como Navidad o Año Nuevo. En este punto se evidencia que los memes ocupan un espacio dentro de los primeros puestos, y luego temáticas como: muertes de personajes famosos o noticias importantes estuvieron presentes, así como concursos que realizó la marca para generar mayor interactividad con la audiencia.

Dentro de los colores más usados en el 2017, están: el blanco con 34 veces, le sigue el naranja con 33, luego el azul y verde con 32 y 30 veces respectivamente. El rojo obtuvo una cantidad de 26, negro 25, amarillo 19, rosado 12 , y finalmente el marrón, gris y púrpura fueron contabilizados con seis veces cada uno. Reflejando así que la marca no se identifica con un color en específico para realizar publicaciones, sino que éste varía según el tipo del mensaje que se difunde.

En cuanto a la ubicación del logotipo la parte inferior izquierda en 45 publicaciones sigue siendo la preferida para ubicar la marca representativa de la empresa, pero en 22 ocasiones se ubica en el centro de la imagen.

El producto en la parte central de la pieza publicitaria es la opción más usada para las publicaciones de la marca, así como la parte inferior del producto. Esto con la finalidad de generar o captar la mayor atención posible en esa parte del producto. La tipografía de color blanco es la usada en los productos, con la finalidad que resalten entre los colores del fondo. Se puede apreciar que se incluyen nuevamente colores como el rosa y el violeta. El verde, el azul, el blanco y el naranja, son los colores con los que se busca que el usuario identifique la marca.

En el 2018 se analizaron 46 publicaciones siendo todas imágenes. Se utilizaron los siguientes hashtags: \#ActitudForestea, \#SinSorbetePorFavor, \#ParadojaSabado, \#Forestea, \#LlénateDeCosasBuenas, \#LlénateDeForestea, y \#Sabiduría. Se obtuvo alrededor de 49.621 "me gusta", 200 "me encanta", 4000 "me divierte", 25 "me asombra", 10 "me entristece" y 6 "me enoja". 
Para el 2018 se ha podido determinar que la mayoría de piezas publicitarias en Facebook se basaron en la temática de promocionar Forestea. Se siguió utilizando la temática de festividades (Navidad, Año Nuevo, etc...). A esto se suma la utilización de memes para poder llegar a un público más amplio en esta red social.

El color rojo fue el que tuvo prioridad al momento de difundir mensajes por parte de la empresa. También los colores blanco y azul se llevan parte de ese protagonismo. Colores que desde sus comienzos con el marketing digital se empezaron a utilizar. Se ha determinado que su utilización es muy variada y mantienen cierta uniformidad en cuanto a utilización de los colores.

En cuanto a la ubicación del logotipo, la parte superior izquierda despuntó entre todas las otras opciones, aunque la parte central de los productos publicitarios, también fue muy usado para ubicar la imagen de la marca en las piezas posteadas. En la mayoría de piezas publicitarias se ubicó a la bebida en la parte central del producto con la finalidad de darle ese protagonismo que merece la bebida en relación con la demás información de las publicaciones.

Tabla 2

Número de "me gusta" totales analizados por años en la página de Facebook de Forestea

\begin{tabular}{|c|c|}
\hline Año & No de "Me gusta" $^{\circ}$ \\
\hline 2013 & 133 \\
\hline 2014 & 1104 \\
\hline 2015 & 887 \\
\hline 2016 & 12628 \\
\hline 2017 & 18113 \\
\hline 2018 & 49621 \\
\hline
\end{tabular}

Fuente: Elaboración propia.

\section{Discusión}

Para lograr determinar el tipo de estrategias que utilizó Forestea en su página oficial de Facebook, se realizó un análisis a base de variables cualitativas observables en los productos comunicacionales presentes en la red so- 
cial. Se puede determinar la importancia de una campaña publicitaria eficaz para el posicionamiento de Forestea en Facebook.

Es necesario una variación de contenidos en las publicaciones de la empresa Forestea para refrescar el contenido compartido y generar mayor interacción y retroalimentación con los seguidores de la página. Puesto que la estrategia de la marca se basa en imágenes y no se varía el tipo de publicaciones. Algo que se considera repetitivo y es necesario variar la forma en cómo se emite un mensaje, aunque en todas las publicaciones el mensaje sea el mismo.

Se logró observar que la estrategia digital de Forestea se basa en una campaña publicitaria donde el mensaje principal de los productos comunicativos es acerca de la bebida. También realizan diferentes concursos para sus seguidores y sus interacciones ocasionan los resultados deseados. Esta estrategia busca generar interés y la idea de consumo del producto. De forma que Forestea está cumpliendo de forma correcta el uso de las estrategias que se ven reflejadas en sus publicaciones. Forestea se mantiene con la estrategia digital de seguir generando contenido de valor para sus seguidores y de esta manera practicar un buen uso del marketing digital en su página oficial de Facebook.

Se debe rescatar que Forestea mantiene su éxito digital debido al correcto uso de los aspectos técnicos en cada publicación. Forestea ha venido generando productos con muchas variantes colorimétricas y quizá de esta manera no se ha logrado identificar y fidelizar a los clientes con una gama de colores bien definida.

El uso del hashtag es importante y se lo debería mantener a lo largo de toda la campaña publicitaria. Es una herramienta que sirve para comunicar algo específico. No debería tener muchos cambios. Forestea utiliza esta herramienta bajo diferentes criterios teóricos puesto que poseen varios hashtags y variantes que no generan el mismo mensaje.

\section{Conclusiones}

La mayoría de publicaciones de Forestea en Facebook son imágenes. De manera que no varía el tipo de publicación y la naturaleza de la marca se mantiene con cierta informalidad. Esto genera buenos resultados en los seguidores de Facebook de Forestea. Las reacciones e interacciones en los 
productos comunicativos fueron más en los últimos años. Las publicaciones que están categorizados como memes tienen la mayor cantidad de reacciones e interacciones por parte de los seguidores.

Los recursos técnicos que utiliza la marca en sus publicaciones son planos generales de personas en un contexto donde se evidencia la existencia del producto y primeros planos del producto. Utilizando la iluminación para resaltar al personaje principal y destacándolo del resto de elementos.

La herramienta más utilizada por la marca es el fotomontaje donde se muestra a la horchata Forestea en diferentes escenarios, acompañado del mensaje implícito de consumir la bebida.

El contexto de los productos analizados no varía. El contenido publicado maneja una misma línea persuasiva y el mismo estilo incluyendo el tipo de mensaje. La mayoría del tiempo se visualiza la presencia del nombre de la marca y la existencia del producto.

Los hashtags utilizados por parte de la empresa son muchos y no mantienen relación entre sí. Esta herramienta genera buenos resultados en la retroalimentación de los mensajes. Los colores más usados por la empresa para las publicaciones son: el rojo, verde, azul, negro, y amarillo. Lo que permite un proceso de identificación y apropiación de los seguidores con la marca.

Forestea mantiene una estrategia publicitaria visual muy activa en Facebook en donde se le da protagonismo a la imagen e ilustración. Estrategia que Forestea utiliza para poder llegar de una manera directa a sus clientes y posibles clientes en la red social.

\section{Bibliografía}

Altamirano-Benítez, V., Marín-Gutiérrez, I., \& Ordóñez-González, K. (2018). Comunicación turística 2.0 en Ecuador. Análisis de las empresas públicas y privadas. Revista Latina de Comunicación Social, (73), 633-647. https://doi.org/10.4185/RLCS-2018-1273

Armijos, C., Matailo, A., Bec, N., Salinas, M., Solano, N., Calva, J., Ludeña, C., Larroque, C., \& Vidari, G. (2020). Chemical composition and selective BuChE inhibitory activity of the essential oils from aromatic plants used to prepare the traditional Ecuadorian beverage horchata lojana. Journal of Ethnopharmacology, 263, 113162. https://doi.org/10.1016/j. jep.2020.113162 
Ballestar, M.T., Grau-Carles, P., \& Sainz, J. (2019). Predicting customer quality in ecommerce social networks: a machine learning approach. Review of Managerial Science, 13(3), 589-603. https://doi.org/10.1007/s11846-018-0316-X

Berceruelo, B., De Antonio, A., Dennetiere, A., Díaz, S., García, A., Geijo, F., Gómez, C., Gómez, C., Gonzalo, P., Lázaro, A., Mariñas, A., Miguel, G., Mimoso, M., Nuevo, N., Ortiz, J., Pereira, A., Prados, A., Pulido, J., Rodríguez, A., Rodríguez, S., Sande, P., Soto, P., \& Sotomayor, C. (2017). Estudio de Comunicación. The Coca-Cola Company. Madrid. https://bit.ly/2NijjWc

Boelsen-Robinson, T., Backholer, K., Peeters, A. (2016). Digital marketing of unhealthy foods to Australian children and adolescents. Health Promotion International, 31(3), 523-533. https://doi.org/10.1093/heapro/dav008

Boyeras, A., Tabachnik, I., Marín, C., \& Kaper, F. (2019). Redes sociales: técnicas del marketing digital. Six ediciones.

Broun-Isaac, J.T. (2020). Desafíos constitucionales en torno a la tutela del derecho de acceso a la información y libertad de expresión en la era digital. Anuario de Derecho Constitucional Latinoamericano, (26), 749-771. https://bit.ly/2PWZJ4r

Camejo, D., Peña, B., \& Valera, M.E. (2020). Plan publicitario para el impulso de las ventas a través de las redes sociales. Revista Peruana de Administración, 2(2), 80-91. https://bit.ly/31MMmXf

Cardozo-Valesca, S.V. (2007). La comunicación en el Marketing. Visión gerencial, (2), 196-206. https://bit.ly/3fFSpFq

Castaño-Garrido, C.M., \& Quecedo-Lecanda M.R. (2003). Introducción a la metodología de investigación cualitativa. Revista de Psicodidáctica, (14), 5-40. https://bit.ly/3mhHMd7

Días, L., Torruco, U., Martínez, M., \& Varela, M. (2013). La entrevista, recurso flexible y dinámico. Investigación en educación médica, 2(7) 162 -167. https://bit.ly/2PzxLvN

Filippone, G., Laganà, V.R., Di Gregorio, D., Nicolosi, A. (2021). Collective and commercial catering services of the ho.re.ca channel: A case study in Calabria (Italy). Smart Innovation, Systems and Technologies (SIST), 178, 823-833. https://doi.org/10.1007/978-3-030-48279-4_77

Gálvez-Clavijo, I. (2016). Facebook para empresas y emprendedores. IC Editorial. García, I. (2011). Marketing Digital Multimedia: Nuevos formatos y Tendencias. https://bit.ly/2GxkcoD

Gómez-Carreño, E.A., \& Palacios-Alvarado, W. (2021). Revisión de literatura sobre Marketing en Redes Sociales. Interfaces, 4(1), 1-16. https://bit.ly/3rRzWb7 
Guevara, M., Proaño, A., Tejera, E., Ballesteros, I., Sánchez, M.E., Granda-Albuja, M.G., Freire, B., Chisaguano, A.M., Debut, A., Vizuete, K., SantosBuelga, C., González-Paramás, A.M., Battino, M., Álvarez-Suárez, J.M. (2020). Protective effect of the medicinal herb infusion "horchata" against oxidative damage in cigarette smokers: An ex vivo study. Food and Chemical Toxicology, 143, 111538. https://doi.org/10.1016/j.fct.2020.111538

Guevara, M., Tejera, E., Iturralde, G.A., Jaramillo-Vivanco, T., Granda-Albuja, M.G., Granja-Albuja, S., Santos-Buelga, C., González-Paramás, A.M., \& Álvarez-Suárez, J.M. (2019). Anti-inflammatory effect of the medicinal herbal mixture infusion, Horchata, from southern Ecuador against LPSinduced cytotoxic damage in RAW 264.7 macrophages. Food and Chemical Toxicology, 131, 110594. https://doi.org/10.1016/j.fct.2019.110594

Hütt-Herrera, H. (2012). Las redes sociales: Una nueva herramienta de difusión, Revista Reflexiones, 91(2), 121-128. https://bit.ly/3ujA6tF

Jackler, R.K., Li, V.Y., Cardiff, R.A.L., \& Ramamurthi, D. (2019). Promotion of tobacco products on Facebook: Policy versus practice. Tobacco Control, 28(1), 67-73. https://doi.org/10.1136/tobaccocontrol-2017-054175

Jiménez, R. (2016). El uso de Facebook como plataforma de mercadeo digital, para la empresa Planeta Urbano de la ciudad de Loja. Universidad Nacional de Loja. https://bit.ly/303MO1W

Jiménez-Marín, G., \& Pérez-Curiel, C. (2021). Las redes sociales como herramienta de comunicación entre públicos: endorsement marketing como forma de publicidad. En Rodrigo Elías Zambrano y Gloria Jiménez-Marín (Ed.), Reflexiones en torno a la comunicación organizacional, la publicidad y el audiovisual desde una perspectiva multidisciplinar (pp. 13-25). Fragua. https://bit.ly/31GXllc

Jumbo, P.P. (2019). Plan de exportación para ampliar la comercialización de la empresa Induloja de la bebida Forestea en Popayán (Bachelor's thesis). Universidad de Otavalo. https://bit.ly/31WTWip

Kotler, P., \& Armstrong, G. (2003). Marketing: versión para Latinoamérica. Pearson. https://bit.ly/2RfiJr7

Lara-Navarra, P., López-Borrull, A., Sánchez-Navarro, J., \& Yánez, P. (2018). Medición de la influencia de usuarios en redes sociales: propuesta Social Engagement. El profesional de la información (EPI), 27(4), 899-908. https://bit.ly/3cOmkZY

Linares-Cazola, J.G., \& Pozzo-Rezcala, S.K. (2018). Las redes sociales como herramienta del marketing relacional y la fidelización de clientes. Universidad César Vallejo. https://bit.ly/3wtkKo6 
López-Jáuregui, Á., Martos-Partal, M., \& Labeaga, J.M. (2019). Impact of SMEs strategy on loyalty: the hairdresser case. Spanish Journal of MarketingESIC, 23(2), 273-293. https://doi.org/10.1108/SJME-11-2018-0051

Marín-Gutiérrez, I. (2020). Métodos y técnicas de la investigación. EdiLoja.

Marín-Gutiérrez, I., Andrade-Vargas, L., \& Iriarte-Solano, M. (2016). Diseño de proyectos de investigación-desarrollo y propuestas metodológicas. Universidad Técnica Particular de Loja.

Matassi, M., \& Boczkowski, P. J. (2020). Redes sociales en Iberoamérica. El profesional de la información (EPI), 29(1). https://bit.ly/3sI8iPf

Monferrer-Tirado, D. (2013). Fundamentos del marketing. Castellón de la Plana: Universitat Jaume I. http://dx.doi.org/10.6035/Sapientia74

Peña, L.B. (2010). La revisión bibliográfica. Universidad Pontificia Javeriana. https://bit.ly/3mheZoU

Pérez-Dasilva, J., Genaut-Arratibel, A., Meso-Ayerdi, K., Mendiguren-Galdospin, T., Marauri- Castillo, Í., Iturregui-Mardaras, L., Rodríguez González, M.M. \& Rivero Santamarina, D. (2013). Las empresas en Facebook y Twitter. Situación actual y estrategias comunicativas. Revista Latina de comunicación social, (68), 676-695. https://doi.org/10.4185/RLCS2013-996

Piatykop, O., \& Pronina, O. (2020). Model selection of the target audience in social networks in order to promote the product. CEUR Workshop Proceedings, 2604, 396-406. https://bit.ly/3uikhDt

Pita-Fernández, S., \& Pértegas-Díaz, S. (2002). Investigación cuantitativa y cualitativa. Cadernos de Atención Primaria, 9 (2), 76-78. https://bit. ly/3mmRDhJ

Ríos, M., Tinitana, F., Jarrín, P., Donoso, N., \& Romero-Benavides, J.C. (2017). "Horchata" drink in Southern Ecuador: medicinal plants and people's wellbeing. Journal of Ethnobiology and Ethnomedicine. https://doi. org/10.1186/s13002-017-0145-z

Selman, H. (2017). Marketing digital. Ibukku.

Sforzin, V. (2016). Redes sociales en Latinoamérica. De los usos a las estrategias colectivas. IX Jornadas de Sociología de la UNLP, 5 al 7 de diciembre de 2016, Ensenada, Argentina. En Memoria Académica. Buenos Aires: https://bit.ly/36EgTYo

Tarro, L., Aceves-Martins, M., Papell-Garcia, I., Arola, L., Giralt, M., Llauradó, E., \& Solà, R. (2017). A youth-led, social marketing intervention run by adolescents to encourage healthy lifestyles among younger school peers 
(EYTO-kids project): A protocol for pilot cluster randomized controlled trial (Spain). International Journal of Environmental Research and Public Health, 14(8), 923. https://doi.org/10.3390/ijerph14080923

Tran, V.T. (2021). Exhibiting Coca-Cola at universal exhibitions. Food, Culture and Society. https://doi.org/10.1080/15528014.2021.1873036

Zeler-Pighin, I. (2017). Facebook como instrumento de comunicación en las empresas de América Latina (Tesis doctoral). Universitat Rovira i Virgili. https://bit.ly/3rInCdq 